\title{
The Collegiate Trainees' Committee position on the use of logbooks in training
}

\author{
Gary Sullivan, Peter Cornwall, Irene Cormac and Maria Atkins
}

The Calman report (Calman, 1993) aimed to reduce the period of postgraduate specialist training to about seven years, introduce the Certificate of Completion of Specialist Training (CCST), reduce the number of training grades to two, and introduce more structured training. Calman also recommended four themes which should guide postgraduate training: flexibility, choice, competency and assessment on merit.

Following the Calman report, and after considerable debate, the CTC agreed to support the establishment of a College Working Party on logbooks, at the request of the Education Committee. This working party was to supervise an 18month evaluation of a pilot logbook or 'personal training file' which can be used by general professional trainees as a means of facilitating the recording and planning of their training in psychiatry. CTC attached considerable importance to this Working Party, and a past chairman of CTC (Dr Annie Hall) was a member. The Working Party reported in October, 1996.

CTC agreed to this pilot of logbooks, provided that the logbook remains the property of the trainee. The CTC regards logbooks in general professional training (GPT) as a means of assessing training rather than trainees.

More recently the Joint Committee for Higher Professional Training (JCHPT: the committee which has responsibility for all aspects of higher training) has been considering a 'Research and Study Logbook for Specialist Registrars in Psychiatry and has produced a draft version.

The CTC considered these matters at its annual residential meeting in September, 1996, attended by the Dean. This report is a result of those deliberations and of further discussions following the report of the logbook working party. and is an attempt to clarify the views of CTC on the use of logbooks in training.

\section{General professional training}

The CTC has previously agreed to a pilot of the use of logbooks in GPT, provided that the logbook remains the property of the trainee.
The Royal College of Psychiatrists has conducted a pilot study of a logbook, and the results are awaited. Some training schemes have used locally designed logbooks for several years already. Several members of CTC have had experience of using these logbooks, and they generally described an initial enthusiasm which became tempered with indifference. This was attributed to a lack of perceived benefits to encourage their completion.

The CTC is opposed to the use of logbooks as a means of summative assessment in GPT since the membership examinations already fulfil this role. We envisage the logbook being put to practical use: (1) in weekly consultant supervision: (2) at the 3 and 6 month appraisal with the clinical tutor; and (3) to guide trainees and clinical tutors as to progress towards eligibility for the examinations. The CTC remains concerned that appointment panels for specialist registrar posts may request to see a candidate's logbook, and that this would disadvantage some candidates due to regional variations in the nature of training.

A logbook may be useful as a means of highlighting deficiencies in an individual's training within a scheme, in order to encourage the tutor to rectify them. They may be useful in informing a trainee at the beginning of training about the various aspects of training which need to be addressed. Logbooks may also be useful in assessing deficiencies in a particular scheme during visits by the Central Approval Panel, leading to improved standards of training.

\section{Higher specialist training}

Many of the arguments for logbooks in GPT also appear to apply to higher specialist training (HST), such as clarification of the aims of training, leverage in terms of securing resources for training, and standardisation of training across different rotations. However, CTC's view is that these points are adequately covered by the current version of the Record of In-Training Assessment (RITA). This form is closely based 
on the College's Educational Policy and covers the necessary curriculum activities (apart from special interest and research). A logbook in addition to RITA (and annual appraisals) would be duplicative and unnecessary. CTC appreciates the efforts made by JCHPT on our behalf, and believes that RITA and the continued vigilance of JCHPT will provide adequate leverage for the provision of quality in training.

Although the CTC has previously criticised a lack of structure in the training received by senior registrars (Davies et al, 1995), we believe that this has been addressed by RITA. CTC values greatly the flexibility in HST which allows Specialist Registrars to undertake research, develop a high degree of expertise in areas of special interest, and undertake training in other areas such as management or teaching. CTC is concerned that a detailed logbook would be more prescriptive and so impair HST by reducing flexibility.

For these reasons, the CTC is opposed to the extension of 'GPT-type' logbooks to HST.

\section{Assessment of research training}

The College Educational Policy of April 1996 outlines the requirements for training in research during GPT and HST. The requirements for GPT cover the knowledge base (principles of scientific reasoning, statistical methods and literature evaluation) and the acquisition of skills and attitudes (the value of critical reading and research skills). This will be done through attending a MRCPsych course, individual consultant supervision and attending courses in research methodology. The requirements do not include pursuing a research project. The requirements for HST incorporate the ability to design, undertake and complete a research project, including the ability to pose relevant research questions, formulate hypotheses, design projects, make statistical evaluations and draw valid conclusions. In the field of audit, trainees in HST will acquire the ability to apply audit principles to their work, to team practice and in a servicewide context.

The GPT logbook currently recommended by the Royal College of Psychiatrists has a significant section on research experience, including a programme for a Masters dissertation and other involvement in active research. Many trainees attend a Masters degree course to develop research skills, but these can be quite demanding. It may be more appropriate for study for a Masters degree to take place during HST.

Although RITA makes only brief reference to assessment of the research skills required during HST, higher trainees get one day per week protected from clinical commitments to be able to undertake and complete a research project.
The CTC believes that trainees should be accountable for how they make use of their protected study time and be able to demonstrate that they are developing the necessary skills at the time of the annual appraisal. The view of CTC is that this could be done by appending a copy of a study plan to RITA. Those higher trainees who elect to pursue more specialised training in research leading to a Masters degree or Doctorate as part of HST would find a research logbook of the kind proposed by JCHPT to be a useful and appropriate tool to assess progress through study for a higher degree.

CTC is concerned that not all trainees are interested in, or successful at, research and they may wish to use the protected time to pursue further training in alternative skills, such as management or teaching. We propose that this should be recognised as a valid and valuable use of protected time if it leads to publication and/or a higher qualification. It has been the consistent view of CTC that not all trainees in HST need participate in original research (Davies et al, 1995). Progress in the study plan could be assessed by the RITA procedure. This would maintain the considerable flexibility in HST.

\section{Acknowledgement}

The authors would like to thank all the members of CTC for their contribution to the working parties, and the further discussions, which made this report possible.

\section{References}

Calman, K. C. (1993) Hospital Doctors: Training for the Future. The Report of the Working Group on Specialist Medical Training. London: HMSO.

Davies, S., Davison, P., SharP, C., et al (1995) Collegiate Trainees Committee Position on Structured Training. Psychiatric Bulletin, 19, 455-458.

*Gary Sullivan, Senior Registrar, Former Chairman, CTC, Department of Psychological Medicine, University of Wales College of Medicine, Whitchurch Hospital, Cardiff CF4 7XB; Peter L. Cornwall, Chairman CTC and Clinical Research Associate, University Department of Psychiatry. The Royal Victoria Infirmary, Queen Victoria Road, Newcastle upon Tyne, NE1 4LP, Irene D. Cormac, Vice-Chairman CTC and Registrar in Psychiatry, South Warwickshire Mental Health Services, Psychotherapy Service, Yew Tree House, 87 Radford Rd, Leamington Spa, CV31 1JQ; Maria Atkins, Honorary Secretary CTC and Senior Registar in Psychiatry. The Woodlands Centre, Hillingdon Hospital, Pield Heath Rd, Uxbridge, Middlesex UB8 3NN

*Correspondence 